\title{
2. Die Eingrenzung des Gebiets
}

Eine definitive Eingrenzung antiker Landschaften erscheint vielfach unscharf und willkürlich, wenn sie sich an den historischem Wandel unterworfenen Provinzgrenzen orientiert, denn hierbei wird der Versuch gemacht, divergierende Angaben aus verschiedenen Epochen miteinander zu harmonisieren.' Gerade in Bezug auf Isaurien waren nicht nur die administrativen Grenzverläufe schwankend, sondern es wurde das isaurische Kernland selbst nach den großen Aufständen in der Spätantike mehrfach aufgeteilt. Bereits Kilikien war nicht genau einzugrenzen. ${ }^{2}$ Die zur Landschaft Lykaonien gehörende Gegend um die Städte Isaura Nova und Vetus gehörte in augusteischer Zeit zum Reich des galatischen Klientelkönigs Amyntas, dann zur Eparchie Lykaonien, nach Gordian III. und Diocletian zur spätantiken Provinz Isauria, schließlich seit Valens wieder zur nördlich angrenzenden Provinz Lycaonia. ${ }^{3}$ Auch auf die antiken Autoren ist für eine genauere Bestimmung des Gebiets nicht immer Verlaß: So ist etwa die im Motto dieses Kapitels angeführte Anmerkung des älteren Plinius, daß Isaurien zu seiner Zeit bis zur Küste gereicht habe, falsch. ${ }^{4}$ Plinius beklagt, daß die Autoren vor ihm Pamphylien mit Kilikien verbunden hätten und das Volk der Isaurier dabei außer Acht gelassen haben. Zumindest bezüglich der Verwaltungsgrenzen kritisiert Plinius seine Vorgänger zu Unrecht. Denn Strabo, den Plinius nicht kannte, nennt nach den Homonadeis die Stadt Coracesium weit westlich von Anemurium an der Grenze zu Pamphylien, was bei Plinius bereits zu Isaurien gehört, die

$1 \quad \mathrm{Zu}$ diesem Problem vgl. Brandt (1992) 8 mit Hinweis auf Isaurien. - Historische Karten Isauriens finden sich in BM II (Anhang); MITFORD (1980) 1260 (hier im Anhang abgedruckt); TAVO 8 (1984), B VI 1; MUTAFIAN (1988) II Karten 8 u. 9: Straßen und Ortschaften; TIB 5,2 (Kartenteil): antike und moderne Ortschaften in Kilikien und Isaurien; Übersichtskarte mit Höhenreliefs, Ortschaften und Straßenverläufen in Quaderni storici 76 (1991) (Tafeln); LENSKI (1999a) 414. (1999b) 312 hat von der geographischen Abteilung der University of Colorado at Boulder eine kleine Karte von hervorragender Übersichtlichkeit mit Höhenrelief, Ortschaften und Straßen erstellen lassen. Die Provinzgrenzen bei Mitchell (1995b) $156 \mathrm{u}$. Treadgold (1995) 12. Die Orte der Kampagne des Servilius sind eingezeichnet auf dem Kleinasien-Blatt bei HAMMOND (1981) pl. 26 a. Zu den Grenzen allgemein MAGiE (1950) I 266-270; Mitford (1980) 1232 ff.; MutafiaN (1988) 14-20. 18.

2 Vgl. z. B. Mitchell (2000) 125: „Cilicia was a notoriously vague geographical term, whose significance varied drastically according to the date or standpoint of the person who used it. Moreover the Cilicians did not form their own koinon until the later years of Hadrian."

3 Zur Verwaltungsgeschichte s.u. im folgenden Kapitel.

4 Vollständig Plin. n.h. V 94: Ciliciae Pamphyliam omnes iunxere neglecta gente Isaurica. Oppida eius intus Isaura, Clibanus, Lalasis; decurrit autem ad mare Anemuri e regione supra dicti. Simili modo omnibus, qui eadem composuere, ignorata est contermina illi gens Omanadum, quorum intus oppidum Omana. Cetera castella XLIIII inter asperas convalles latent. 
,westliche Festung von Kilikien“. Kilikien hat sich somit zu Plinius' Zeit durchgängig an der Küste bis nach Pamphylien hingezogen. Wohl aufgrund der Nachricht des Plinius hat sich der Irrtum verfestigt, daß die Isaurier sich bereits zu dieser frühen Zeit als Piraten betätigt hätten und ihre Heimat sich bis zur Küste erstreckt habe. Die Stelle ist wohl am ehesten erklärbar durch eine vorübergehende Vereinigung des eigentlichen Isaurien mit einem Teil des Rauhen Kilikien zwischen 39 und 36 v. Chr., als Marcus Antonius dem Klientelkönig Polemo Teile von Kilikien gab. ${ }^{5}$ Auch mag er vielleicht von der bei Sallust geschilderten Kampagne des Publius Servilius Vatia ausgegangen sein, der von Pamphylien aus von Norden kommend nach Isaurien vorgedrungen ist. Isaurien bildet in der Tat nördlich des Taurusmassivs dann eine Linie mit Pamphylien und Kilikien. Auch bezieht sich Plinius in dieser Stelle ausdrücklich auf das Volk der Isaurier, nicht auf die Landschaft.

Aus den erwähnten Inkonsistenzen folgt, daß aus pragmatischen Gründen - der Focus dieser Arbeit liegt auf Isaurien in der Spätantike - eine zumindest ungefähre Eingrenzung des Gebiets unumgänglich ist. Isaurien läßt sich im Groben zunächst in west-östlicher Richtung als das Gebiet zwischen dem Trogitis-See und den Flüssen Melas (Manavgat) und Kalykadnos (Gök Su, sowohl dessen Nord- als auch Südarm) einschließlich dem nördlich gelegenen Siedlungsraum der Homonadeis zwischen Trogitis- und Caralis-See definieren. ${ }^{6}$ Dazu kam in der Spätantike seit der Schaffung der Provinz Isauria durch Diocletian die östlich gelegene Ketis, die flache und daher leicht zugängliche Landschaft der Kieten im Land um Olba mit den kleineren Landschaften Kennatis, Lakanitis und Lalassis hinzu. ${ }^{7}$ Die Grenze nach Westen zur Nachbarprovinz Pamphylia et Pisidia lag im Hügelland zwischen dem heutigen Domalani und Aidap. Irgendwann in der frühen Kaiserzeit hatte sich die Grenze nach Osten verschoben, sie befand sich im 2 . Jahrhundert $\mathrm{n}$. Chr. zwischen den Ortschaften LaAnm. 27 Diskussion der älteren Forschung zu den Quellen des Plinius, die eine offizielle Quelle vermutet. Problematisch bleibt wiederum die Grenzlinie der Provinz Asia zu dieser Zeit; vgl. u. Kap. II.1.2. Ein Stück des westlichen Rauhen Kilikien mit einem Zugang zum Meer könnte sogar noch zur Augusteischen Galatia gehört haben, so JONES ( $\left.{ }^{2} 1971\right) 214 f$. 438 nach Ptol. V 5, 3f., der das Küstenland des Rauhen Kilikien unter Pamphylien ein-

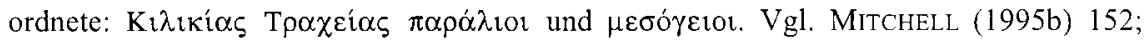
SYME / BIRLEY (1995) 219, Anm. 26. 240.

6 BM II 2, Anm. 21; TIB 5,1 17, 27; allgemein Mutafian (1988) 14 ff.; Syme (1987) 142145, gefolgt von LENSKI (1999a) 431f., Anm. 92.

7 RAMSAY (1890) 364; TIB 5,1 301 s.v. 'Kêtis'. BM I, Anm. 2 schließen aus ihrer isaurischen Inschriftensammlung aus: Seleucia, da schon in MAMA III aufgenommen; Mut (Claudiopolis), Sinapic (Dalisandus) und Dağ Pazarı (Coropissus), um die Einheit der Landschaft Ketis zu bewahren. Die Landschaft war auch in der Antike geographisch nur schwer zu umreißen, vgl. GotTER (2001) 290, Anm. 13. 
ërtes (Çebel Ires) und Syedra an der Küste; letzteres gehörte bereits zur Pamphylia. ${ }^{8}$ Hier hatte sich wohl auch die westliche Grenze des Königreichs des Amyntas während der späten Republik befunden. Nach Osten war die Provinz im Landesinneren durch den Antitaurus, an der Küste wohl durch den Kalykadnos begrenzt, denn die unweit östlich gelegenen Küstenstädte Korykos, Elaiussa-Sebaste und Pompeiopolis (zuvor Soloi) gehörten schon zur spätantiken Cilicia Secunda. ${ }^{9}$

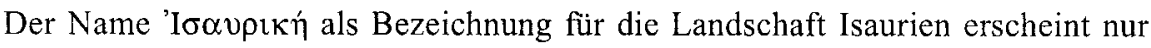
bei den kaiserzeitlichen Autoren Strabo und Plinius, nicht mehr später, da sich diese dann mit der Verwaltungseinheit Isauria deckte. Diese wurde Teil der im 2. Jahrhundert n. Chr. neugeschaffenen Provinz der Drei Eparchien Kilikien, Isaurien und Lykaonien, während der alte Name lediglich eine Landschaft bezeichnet hatte. Für die Zeit vor der neuen Provinz ist also die Bezeichnung „Isauria“ entweder ein Kopistenfehler, wie bei Strabo, oder ein Anachronismus. ${ }^{10}$ Wenn in dieser auf die Spätantike fokussierten Arbeit also von „Isaurien“ die Rede ist, so ist damit das Gebiet gemeint, welches sich mit der Provinz Isauria sowie dem zur Lycaonia gehörigen Streifen am nördlichen Taurushang vom Trogitis-See bis Laranda deckt, in welchem sich die drei mit Isaura bzw. Isauropolis benannten Städte befanden.

8 Jones ( ${ }^{2} 1971$ ) 541, Appendix 4 mit den Bischofslisten. Plin. n.h. V 93 nennt im 1. Jh. n. Chr. den westlich gelegenen Melas die alte Grenze, finis antiquus Ciliciae Melas amnis; und Ptol. V 5, 3 vermerkt Mitte des 2. Jahrhunderts Syedra als eine Küstenstadt des Rauhen Kilikien in Pamphylien; vgl. TIB 5,1 17; ERZEN (1940) 76. Dafür gibt es auch ein archäologisches Zeugnis: Hans-Jörg KELLNER, 'Zwei neue Flottendiplome zur Grenze von Pamphylien und Kilikien' in: Chiron 7 (1977), 315-322. 320f. für 139/145 n. Chr. Steph. Byz. Ethnika p. $589=F H G$ IV p. 134 erwähnt ein Fragment des isaurischen Historikers Capito Lycus, wonach Syedra im 6. Jh. n. Chr. eine isaurische Stadt gewesen sei; nach ELTON (2000a) 295 ist damit jedoch die Landschaft Isaurien gemeint, die von der Provinz zu unterscheiden sei. Russell (1991) 487 vermutet plausibel den $7 \mathrm{~km}$ östlich von Cebel Ires gelegenen Sedra Çay als Grenze zwischen der kaiserzeitlichen Pamphylia und Cilicia. Diese Grenze dürfte dann für die Isauria beibehalten worden sein.

9 Ramsay (1890) 383, nach der Teilnehmerliste des Chalkedonense im Jahr 451. Somit bildet auch nicht erst Pompeiopolis die östliche Grenze, wie von TIB 5,1 381 s.v. 'Pompeiupolis' aufgrund von Pereg. Aeth. XXIII 1 u. Hierocl. 704, 3 angenommen.

10 Ramsay (1941) 231. 232, Anm. 3 zu Strab. XII 6, 3 vergleicht mit der Landschaft Пıбı-

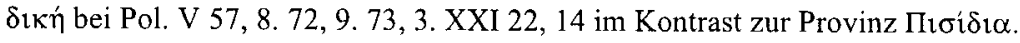

